ISSN: 2600-5859

WwW.concienciadigital.org

Vol. 3, $\mathrm{N}^{\circ} 1.1$, p. 29-43, febrero, 2020

\title{
Diseño y selección de cuchillas para el proceso de trituración de neumáticos y análisis de costos de fabricación
}

\author{
Design and selection of blades for the tire crushing process and \\ manufacturing cost analysis.
}

Javier Renato Moyano Arévalo. ${ }^{1}$, Eugenia Mercedes Naranjo Vargas. $^{2}$ \& Alex Giovanny Tenicota García. ${ }^{3}$,

Recibido: 10-12-2019 / Revisado: 26-12-219 /Aceptado: 04-01-2020/ Publicado: 07-02-2019

\begin{abstract}
.
DOI: $\underline{\text { https://doi.org/10.33262/concienciadigital.v3i1.1.1130 }}$

For the reuse of vehicle tires, a process called crushing is presented, which allows recycling and use of different materials for use in various industrial applications. The basic design of the tire crushing system using efficient blades was considered as an alternative solution to the problem of several business sectors, which have not yet reused the material. The objective of the research pursues the design and selection of blades for the tire crushing process and manufacturing cost analysis. Therefore, typical mechanical properties of rubber in the cutting process were analyzed, and economic knife alternatives were compared for the establishment of an appropriate tire crushing process. Each of the alternatives compared differs in mechanical characteristics and nature of the material widely spread in the local environment such as ASTM-A36, SAE -1045, and SAE-S1, the first material being the most economical and recommended, as long as the design alternative is used according to the curved flank model, with maximum deformation on average of $0.0202 \mathrm{~mm}$. It concludes with a low manufacturing cost regardless of the design of the blade varies, in any case heat treatment must be performed. The crushing system was sized in such a way that untreated tires are treated, typical in small vehicles, because they are very numerous since there are fewer cases

\footnotetext{
${ }^{1}$ Escuela Superior Politécnica de Chimborazo, Facultad de Mecánica, Carrera de Ingeniería de Mantenimiento Industrial. Grupo de Investigación Ciencia del Mantenimiento CIMANT. Riobamba, Ecuador, javier.moyano@espoch.edu.ec

${ }^{2}$ Escuela Superior Politécnica de Chimborazo, Facultad de Mecánica, Carrera de Ingeniería Industrial. Grupo de Investigación de Energía, Ambiente y Productividad ENAMPROD Riobamba, Ecuador, eugenia.naranjo@espoch.edu.ec

${ }^{3}$ Escuela Superior Politécnica de Chimborazo, Facultad de Mecánica, Carrera de Ingeniería de Mantenimiento, Grupo de Investigación del Mantenimiento GIMAN. Riobamba, Ecuador, alex.tenicota@espoch.edu.ec
} 
of retreading. The research becomes a sustainable and feasible alternative for the medium and small industry that is good at offering products based on recycled material such as rubber.

Keywords: Tire Reuse, Crushing Process, Mechanical Properties, Manufacturing Costs

\section{Resumen.}

Para la reutilización de los neumáticos de vehículos, se presenta un proceso llamado trituración, el cual permite reciclar y aprovechar los distintos materiales para uso de diversas aplicaciones industriales. El diseño básico del sistema de trituración de neumáticos mediante cuchillas eficientes fue considerado como alternativa de solución a la problemática de varios sectores empresariales, que aún no han reutilizado el material. El objetivo de la investigación persigue el diseño y selección de cuchillas para el proceso de trituración de neumáticos y análisis de costos de fabricación. Por consiguiente, se analizaron propiedades mecánicas típicas del caucho en el proceso de corte, y fueron comparadas las alternativas económicas de cuchillas para el establecimiento de un proceso adecuado de trituración de neumáticos. Cada una de las alternativas comparadas difiere en características mecánicas y naturaleza del material muy difundidos en el medio local como el caso de ASTM- A36, SAE -1045, y SAE- S1, siendo el primer material el más económico y recomendado, siempre y cuando se utilice la alternativa de diseño según el modelo de flancos curvos, con deformación máxima en promedio de $0.0202 \mathrm{~mm}$. Se concluye con un costo de fabricación bajo independientemente que varíe el diseño de la cuchilla, en todo caso se debe realizar tratamiento térmico. El sistema de trituración fue dimensionado de tal manera que se traten neumáticos desalambrados, típicos en vehículos pequeños, por ser muy numerosos ya que existen menos casos de reencauche. La investigación se convierte en una alternativa sustentable y factible para la industria mediana y pequeña que tiene a bien ofertar productos a base de material reciclado como el caucho.

Palabras clave: Reutilización de Neumáticos, Proceso de Trituración, Propiedades mecánicas, Costos de Fabricación

\section{Introducción}

Los neumáticos de caucho usados en los vehículos son materiales que deben reutilizarse, sin embargo, el incorrecto tratamiento que se le da como residuos ha definido, que, luego de un desgaste excesivo es imposible reutilizarlos para un reencauche o renovación superficial. Según el Ministerio de Ambiente Ecuatoriano; entre 2 a 2,5 millones de neumáticos son desechados en Ecuador por año, y al momento son mínimos los planes de manejo ambiental que evidencien acciones en pro de la reducción o reutilización correcta de los materiales. De acuerdo al trabajo experimentado de (Alejandre, 2014), en España se recogieron alrededor 


\section{ISSN: 2600-5859}

de 162.599 toneladas de neumáticos en 2013 con la participación del SIGNUS (Sistema Integrado de Gestión de Neumáticos Usados), cifra que resulto con 75.559 toneladas de caucho fueron trituradas y granuladas para diversas aplicaciones (mezclas asfálticas, losetas de seguridad para parques infantiles, campos de fútbol de césped artificial, y objetos moldeados). Se reconoce a la trituración como proceso que permite reciclar y aprovechar los distintos materiales que componen a los neumáticos, de modo que en su mayor parte provienen del GCR (granulado de caucho reciclado). Un reciclaje sin reutilización en el caso de neumáticos se convierte en un problema de sobreacumulación, el cual es causante de los daños ambientales, se ha manifestado en pequeñas y medianas bodegas o vulcanizadoras que por falta de presupuesto no cuentan con maquinaria capaz de mejorar las condiciones para la disposición y reutilización del caucho de llantas en la actualidad del país.

Distintos investigadores como (Peláez Arroyave, Velásquez Restrepo, \& Giraldo Vásquez, 2017), (Guerrero, 2018), plantean diversas formas de reutilización del caucho reciclado, incluyendo aplicaciones actualmente comercializadas y otras derivadas estudios de ingeniería. Mismos autores concuerdan que varios métodos que tienen el objeto de aprovechar residuos de caucho, son clasificados en procesos mecánicos, químicos, térmicos, criomecánicos, químico-mecánicos, termo-mecánicos, biotecnológicos, procesos con microondas y con ultrasonido. En la mayoría de estos procesos es necesario triturar el caucho hasta reducirlo a gránulos de tamaño y forma regular, y de esa manera facilitar que los aditivos químicos y agentes de expansión reaccionen adecuadamente con la estructura vulcanizada, a la vez que se separan las fibras de acero, textiles y aditamentos metálicos. Las aplicaciones encontradas en los sectores de construcciones civiles, tales como asfaltos, concretos y materiales aislantes, utilizan mayor potencial de volumen consumido. Sea en el caso de la elaboración de bloques de hormigón, el uso de partículas de caucho en diferentes porcentajes de sustituciones por agregado fino, ha suministrado de características técnicas y económicas típicas de un hormigón convencional, cumpliendo la norma NTE INEN 3066 2016-11. "Se comprobó también que la densidad del concreto disminuye al aumentar la cantidad de partículas de neumático y mejora su resistencia.” (C. Flores, 2014)

El diseño básico de un sistema de trituración de neumáticos se convierte en una alternativa de solución a la problemática empresarial con enfoque de proyección industrial. Si no se ha reutilizado el material para la elaboración de nuevas aplicaciones industriales en diversos ámbitos, la industria ha perdido su horizonte. El sistema de trituración de neumáticos se debe dimensionar con la finalidad de tratar los desalambrados, de los cuales el mayor número responde a casos de vehículos pequeños, caracterizados por no recurrir al proceso de reencauche. Con la finalidad de triturar de manera eficiente, se requiere del dimensionamiento y seleccionamiento adecuado de los componentes críticos que formarán parte del mecanismo cuya transmisión sea económicamente sustentable. Los métodos de selección de elementos de máquinas son actualizados en función del avance tecnológico, de tal manera que la informática contribuye con la simulación del movimiento y propiedades 
ISSN: 2600-5859

mecánicas en concordancia de conocimientos de ingeniería se lo haga una realidad. En la selección adecuada de los componentes mecánicos, debe considerarse el uso racional de energía en el proceso de trituración y molienda de materiales, así como lo manifiesta (José Ramón Hechevarría \& Proeza, 2015). Entre el 3 al 4 \% de la energía generada en el mundo, se destina a las operaciones de reducción de tamaño, siendo que en los Estados Unidos se destina el $29,3 \%$ de toda la energía consumida en la minería.

Según (Roberto Pérez Aparicio, Departamento de Desarrollo de Mercados y Nuevas Aplicaciones, 2018) para que se dé la trituración de neumáticos enteros se necesita de una cinta, pala o un pulpo para su transportación y para el trabajo propio de trituración; que contempla entre uno a dos rotores o ejes de cuchillas, las cuales giran en sentido contrario, con la intención de cortar el neumático, y de acuerdo a sus dimensiones y disposición de la criba, deba obtenerse trozos de entre 20 y $400 \mathrm{~mm}$. Sin embargo, el proceso de granulación se convierte en una etapa determinante para el triturado de neumáticos, debido a que los molinos son separados de la totalidad del acero y parte del textil, pero en esencia las cuchillas toman un papel protagónico. Las cuchillas se convierten en el elemento mecánico imprescindible para el proceso, ya que la calidad del mismo funge como determinante para el corte y granulación de los neumáticos. Los equipos que han sido importados con el afán de ser parte del proceso de trituración a nivel local, han manifestado mecanismos que al revisarlos se evidencia una falta de estudio de las cuchillas y sus mecanismos.

El objetivo de la presente investigación persigue el diseño y seleccionamiento de las cuchillas para el proceso de trituración de neumáticos y el análisis de costos de fabricación entre casos de diseño, material y geometría de los filos de corte.

Para ello se debe establecer los parámetros básicos y requerimientos para el triturador, plantear el sistema de transmisión de la adaptación, modelar y simular las características mecánicas básicas mediante la utilización de herramientas CAD. Para establecer los requerimientos básicos del triturador, se considera el estudio del comportamiento ideal del material objeto a trituración, de modo que evidencie la incidencia de la fuerza de tensión, deformación, dureza, resistencia al desgarre, y deformación a la compresión para con la selección de elementos mecánicos que puedan soportar ciertas condiciones de temperatura de forma estandarizada.

Los mecanismos con diseño eficiente, mismos que reúnan características mecánicas superiores a lo convencional, son parte de una alternativa ideal que debiere implementarse de acuerdo a las mejoras en los procesos industriales que persiguen resultados positivos en productividad y calidad. Un proceso de trituración que cuente con partes mecánicas bien dimensionados en geometría, movimiento, características mecánicas, a bajo costo es lo que varios sectores locales de bajos recursos buscan insistentemente. 


\section{Métodos y Materiales}

Para el desarrollo de la presente investigación se desarrolló la determinación de las propiedades mecánicas del caucho y efectividad del corte del material, y la comparación de costos de las alternativas vigentes de cuchillas para el proceso de triturado en el mercado local. En ese sentido se pudo definir un mejor criterio para la selección adecuada alternativas en lo que respecta al diseño de cuchillas y mecanismos del proceso de corte.

\section{Análisis de las propiedades mecánicas del caucho en el proceso de corte}

Mediante investigación exploratoria y análisis de fuentes bibliográficas se determinaron las propiedades mecánicas del caucho, y la efectividad de los tipos de corte del material. Para determinar el tipo de corte más apropiado para realizar la trituración es necesario analizar el material que para nuestro caso es caucho (copolímero estireno-butadieno). Para los cálculos que se realiza el presente proyecto se considera principalmente la zona del neumático con mayor resistencia es decir el lugar donde se encuentra conformado con alambres. La incidencia de la fuerza de tensión fue definida en base de parámetros indicados en la norma ASTM D412. En el mismo sentido la dureza fue tratada mediante los criterios de la ASTM D2240-05.

El caso de la resistencia al desgarre fue mediante ASTM D624-00. La deformación permanente por compresión fue el caso del criterio estandarizado de ASTM D395-14. En lo posterior se concatenó cada una de las incidencias manifestadas por las resistencias mecánicas en estudio para dar con la manifestación de similares condiciones energéticas y temperatura que podrían ser determinantes para la selección de los elementos mecánicos" (Urrego Yepes \& Cardona Vazquez, 2017).

Fue considerado el trabajo de (Stalin, 2017), para la implementación de la trituradora de neumáticos de caucho, la cual obtiene una granulometría fina, y en base a diferentes cálculos para realizar una correcta construcción, así como también basados en la norma ASTM D 422 del ensayo de corte del material, se determinó cuál es el torque necesario para el motor.

Además se tomaron en cuenta los resultados de los ensayos realizados al caucho, y la información que detalla (Pelizzoni, Fumagalli, Quarleri, \& Raùl, 2013), el cual define que la resistencia a la tracción se encuentra en un rango de 1 y $20 \mathrm{MPa}$, dependiendo del grado de vulcanización, concluyendo en un valor inferior a 35 veces del acero componente de los alambres. Se utiliza la norma ASTM D 4-22 para establecer el tamaño de picado del caucho, en la norma establece que se debe emplear tamices en un rango de numeración 4 a 16 que corresponden a diámetros dese $1,18 \mathrm{~mm}$ a $4,75 \mathrm{~mm}$, para la obtención de granulometría.

Comparación de alternativas económicas de cuchillas para el proceso trituración de neumáticos. 
Se inició con la selección de materiales adecuados para la fabricación de las cuchillas de manera que tenga prestaciones en el mercado, y que además sea económicamente accesible, ya que en el actual mercado local se comercializan cuchillas a un alto costo y con materiales difícilmente de adquirir producto de exportaciones innecesarias. En ese sentido mediante método inductivo se estableció la comparación de precio de fabricación para obtener una alternativa para mejorar el proceso de corte del caucho en lo que respecta al elemento cuchilla.

Fue analizado el proceso de corte de distintos materiales con la finalidad de establecer el mejor proceso para realizar el triturado del caucho, luego de analizar distintos procesos se observa que el corte por seccionado es el que más se destaca en cuestión de separar las distintas partes de una pieza sin que se produzca viruta

\section{Resultados y Discusión}

Las propiedades mecánicas evidenciaron alta deformación elástica bajo estrés es decir sin deformación permanente en comparación con materiales similares. Según los estudios del caucho en los trabajos especializados de (Marisol, 2018), se determina que presenta una buena resistencia a la abrasión y al impacto, pero tiene una resistencia moderada a la flexión y al desgarro propia de una referencia en promedio $0,176 \mathrm{Kg} / \mathrm{mm}^{2}$ de resistencia a la rotura. Las propiedades del caucho sirvieron como referencia para establecer un proceso de corte por cizallado, siendo el más recomendado, por desarrollarse en zonas localizadas con fuerzas en sentido trasversal.

Para un correcto análisis se establece condiciones de funcionamiento entre ellos que el corte se realizará en neumáticos rin (13 - 15 pulgadas) con una velocidad de 10 - $15 \mathrm{rpm}$; el sistema de trituración permitirá obtener partes que oscilarán entre 5 a $7 \mathrm{~cm}$ aproximadamente, los parámetros del sistema como, transporte, almacenamiento, fabricación y demás maquinaria del proceso del sistema de trituración no serán tratadas en detalle en este proyecto.

Al realizar el análisis del proceso de corte y de la potencia requerida para el trabajo se establece utilizar una la transmisión por cadena y por engrane, esto se fundamenta en que las dos trasmisiones generan mayor potencia, agarre, eliminando el deslizamiento y suprimiendo las pérdidas de potencia por fricción de elementos, en el momento de realizar el trabajo de trituración

Para obtener una selección adecuada del perfil de la cuchilla de trituración es prioritario obtener la potencia necesaria de trabajo que permita realizar correctamente la actividad, para lo que se considera una granulometría de $60 \mathrm{~mm}^{2}$ de área

\section{Cálculo de la potencia del motor}


ISSN: 2600-5859

Datos:

$$
\begin{aligned}
\omega_{1}=10-15 \mathrm{rpm} \\
\tau \text { caucho }=0.176 \frac{\mathrm{Kgf}}{\mathrm{mm}^{2}} \Rightarrow>1725490.19 \frac{\mathrm{N}}{\mathrm{m}^{2}}
\end{aligned}
$$

Fuerza de corte de la cizalla.

$$
\begin{gathered}
\tau=\frac{F}{A} \\
F=\tau * A \\
F=0.176 \frac{K g f}{\mathrm{~mm}^{2}} *\left(\frac{\pi}{4}\left(160 \mathrm{~mm}^{2}\right)\right) \\
F=497.62 \mathrm{Kg} f=\mathbf{4 8 7 9 . 9 8 5 ~} \mathbf{N}
\end{gathered}
$$

\section{Calculo del volumen y del torque}

\section{Calculo del volumen según el triángulo de cizallas}

$$
\begin{gathered}
\boldsymbol{V}=\frac{\boldsymbol{b} * \boldsymbol{A}}{\mathbf{2}} * \boldsymbol{l} \\
V=\frac{10 \mathrm{~cm} * 6 \mathrm{~cm}}{2} * 90 \mathrm{~cm} \\
V=\mathbf{0 . 0 0 2 7 \mathbf { m } ^ { 3 }}
\end{gathered}
$$

\section{Calculo del torque}

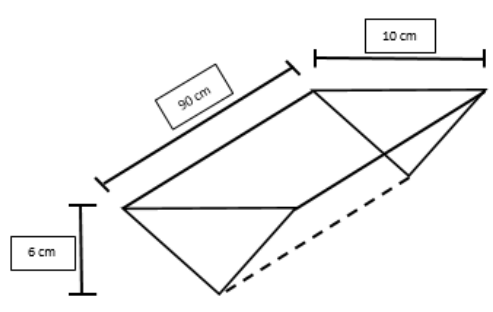

$$
\begin{gathered}
\mathbf{T}=\boldsymbol{\tau} * \boldsymbol{V} \\
\mathrm{T}=1725490.19 \frac{N}{\mathrm{~m}^{2}} *\left(0.0027 \mathrm{~m}^{3}\right) \\
\mathrm{T}=\mathbf{4 6 5 8 . 8} \mathbf{N m}
\end{gathered}
$$

\section{Calculo de la potencia del motor}

$$
\begin{gathered}
\boldsymbol{P}=\boldsymbol{T} \boldsymbol{\omega} \\
P=4658.8 \mathrm{Nm}\left(1570 \frac{\mathrm{rad}}{\mathrm{s}^{2}}\right) \\
P=7318.025 \mathrm{Watt} * \frac{1 \mathrm{Hp}}{746 \mathrm{Watt}}=9.80 \mathrm{Hp} \\
\boldsymbol{P}=\mathbf{9 . 8 0} \mathbf{H p} \approx \mathbf{1 0 ~} \mathbf{H p}
\end{gathered}
$$

Con la finalidad de obtener un correcto selecciona miento de motor, los catálogos sugieren realizar el cálculo previo de una potencia de diseño, que garantice el buen funcionamiento del sistema. Para aseverar que el sistema obtendrá un acertado funcionamiento se debe relacionar el potencia de trabajo con un factor de servicio que garantizara una buena operatividad del sistema 


$$
\begin{gathered}
P_{D}=P_{\text {motor }} * F S \\
P_{D}=10 h p * 1.5 \\
P_{D}=15 h p
\end{gathered}
$$

Luego de obtener la potencia y fuerza de corte a las que estarán sometidas las cuchillas por el corte de los neumáticos, es necesario modelar tres prototipos con distintas configuraciones de cuchillas como se observa en la figura 1. El modelado se realiza mediante software CAD, que sea versátil y de fácil interpretación, estos programas son de gran ayuda en ingeniería ya se puede obtener una apreciación real de los elementos que se requiera representar.

(A)

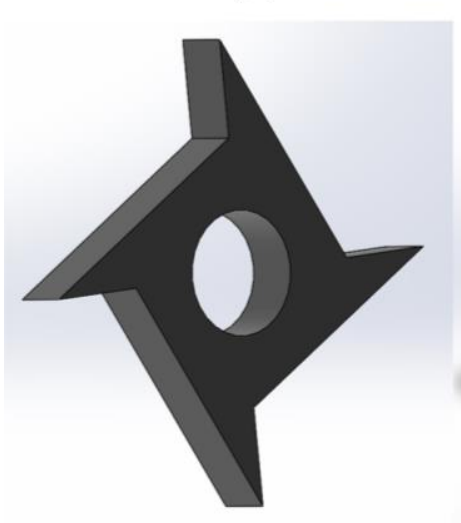

(B)

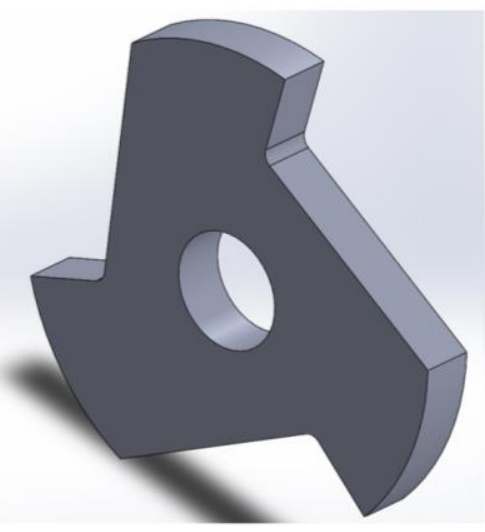

Figura 1. Modelos de cuchillas, Fuente: Autores

En el mercado convencional existen distintos tipos de materiales con los que se puede fabricar las cuchillas, pero cada uno dependiendo de las propiedades tendrá mayor o menor resistencia para realizar el trabajo especificado. Considerando los desgastes prematuros se plantea evitar dichas falencias estableciendo un análisis de los tres diseños de elementos de cortes con distintos materiales existentes en el mercado como son acero ASTM A36 (figura 2), SAE1045 (figura 3), y SAE S1(figura 6). El análisis se lo realiza mediante software de simulación localizando las fuerzas en sectores específicos y de gran incidencia para evidenciar los cambios y afectaciones que se produzcan en los modelos.

Para obtener una certeza en los datos que se van a encontrar, se establece mallados que más se acoplen a nuestros diseños, recordando siempre que del tipo de malla se ajustara los valores reales obtenidos, para nuestro proyecto se establece un mallado con tetraedros, pero así también para obtener los mismos efectos en todos los materiales se alterna el tipo de material con las mismas fuerzas. 


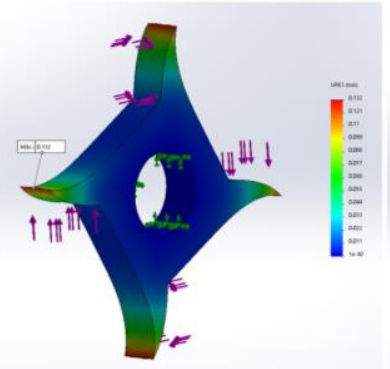

(B)
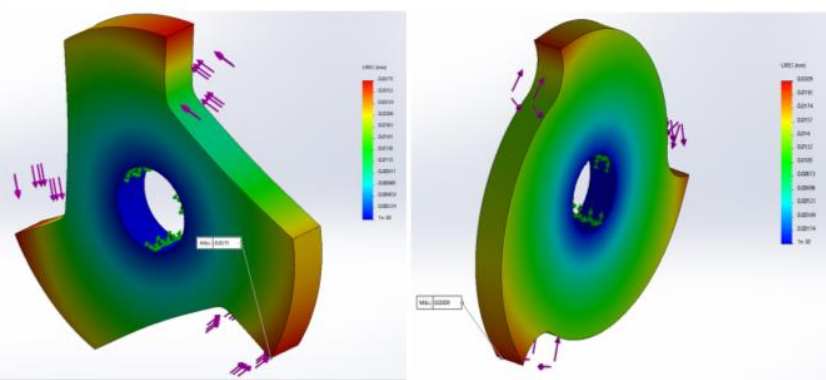

Figura 2. Cuchilla de acero A36. (A) modelo con flancos rectos, con una deformación máxima de $0.132 \mathrm{~mm}$ (B) modelo con flancos a 75 grados con deformación máxima de 0.0275 (C) modelo de flancos curvos, deformación máxima 0.0209 .

Fuente: Autores

(A)

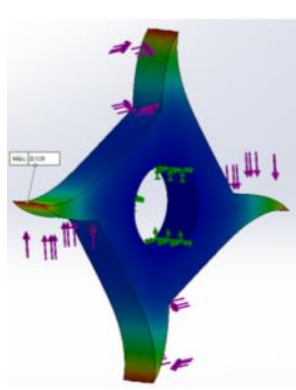

(B)

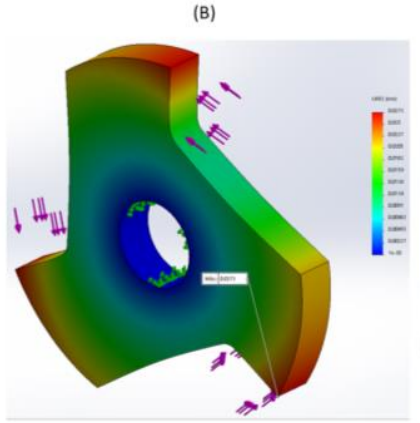

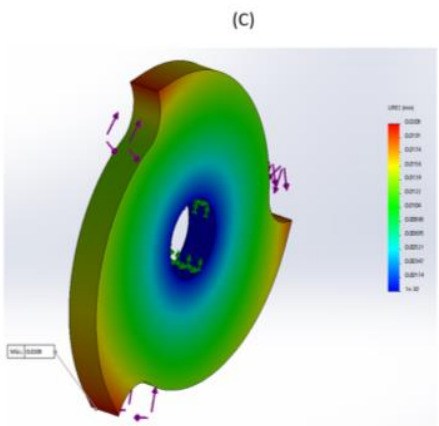

Figura 3. Cuchillas de acero SAE 1045. (A) modelo con flancos rectos, con una deformación máxima de $0.129 \mathrm{~mm}$ (B) modelo con flancos a 75 grados con deformación máxima de 0.0273 (C) modelo de flancos curvos, deformación máxima 0.0208 .

Fuente: Autores

(A)

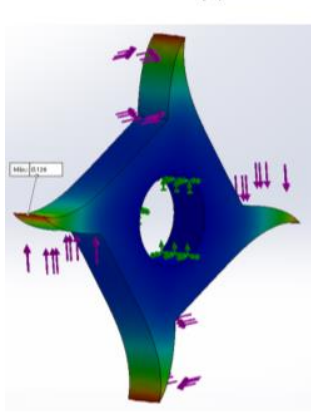

(B)

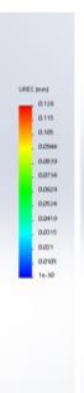

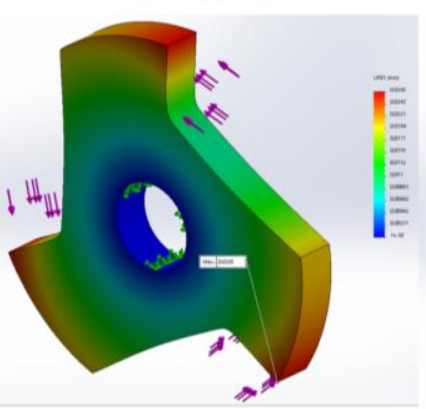

(C)

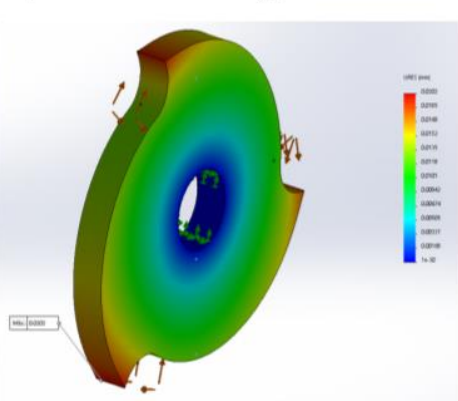

Figura 4. Cuchillas de acero SAE S1. (A) modelo con flancos rectos, con una deformación máxima de $0.126 \mathrm{~mm}$ (B) modelo con flancos a 75 grados con deformación máxima de $0.0265 \mathrm{~mm}$, (C) modelo de flancos curvos, deformación máxima $0.0202 \mathrm{~mm}$.

Fuente: Autores 
ISSN: 2600-5859

El corte por cizallado fue definido el más apropiado entre los vigentes para corte en frío mediante un metal con la presencia de mecanismo de sistema de tijeras (cizalla); el cual presenta un modo de operación normal a la superficie.

En la Figura 5 se observa distintos procesos de corte. El proceso de seccionado corresponde al corte por cincel, este proceso se realiza sin arranque de viruta. El corte por arranque de viruta es un corte donde se usa una cuña y el proceso de cizalla que es un corte tipo tijera.

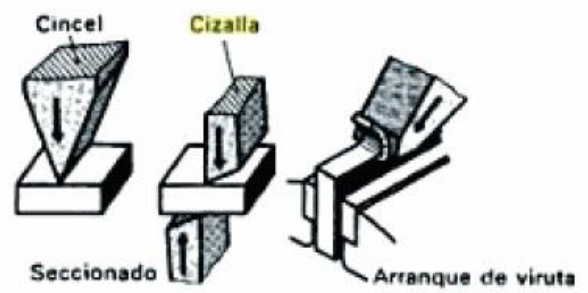

Figura 5: Cortes por cuña/cincel, cizalla y arranque de viruta.

Fuente: (Pelizzoni, 2013)

El proceso de corte mediante arranque de viruta en los metales ocurre cuando la herramienta de corte genera deformación elástica de la parte de metal que se convertirá en viruta, en el transcurso de este proceso se provocan grandes tensiones, hasta que el material supera la tensión por fluencia, produciendo la separación de la capa debido a la deformación plástica. Se considera que el mismo proceso ocurrirá con el caucho

En la Figura 6 (a) se muestra la geometría básica correspondiente a una herramienta de corte y en la 2(b) se detallan los ángulos de la misma respecto al avance del material. De acuerdo a consideraciones técnicas, la suma de los ángulos de incidencia $(\alpha)$, el de filo $(\beta)$ y el de ataque $(\gamma)$ debe ser de $90^{\circ}$.(Pelizzoni, 2013)
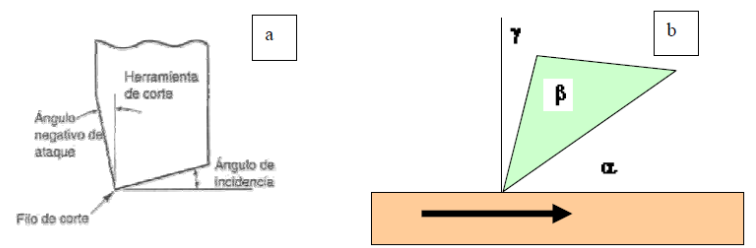

Figura 6: Geometría básica de herramienta de corte por cizalla.

Detalle de los distintos ángulos.

Fuente: (Pelizzoni, 2013)

Para la selección de la cuchilla y material se establece una tabla donde se identifica las propiedades principales e influyentes para la selección 
ISSN: 2600-5859

www.concienciadigital.org

Tabla 1. Costos de fabricación de cuchillas

Vol. 3, $\mathrm{N}^{\circ} 1.1$, p. 29-43, febrero, 2020

\begin{tabular}{|c|c|c|c|c|c|}
\hline \multirow[b]{2}{*}{ Material } & \multirow{2}{*}{$\begin{array}{l}\text { RESISTENCIA } \\
\text { (Mpa) }\end{array}$} & \multicolumn{3}{|c|}{ DEFORMACIÓN (mm) } & \multirow{2}{*}{$\begin{array}{l}\text { COSTO DE } \\
\text { FABRICACIÓN } \\
\text { (Dólares) }\end{array}$} \\
\hline & & A & B & $\mathrm{C}$ & \\
\hline ASTM- A36 & 250 & 0.132 & 0.0275 & 0.0209 & 140 \\
\hline SAE -1045 & 413 & 0.129 & 0.0273 & 0.0208 & 160 \\
\hline SAE- S1 & 680 & 0.126 & 0.0265 & 0.0202 & 215 \\
\hline
\end{tabular}

Fuente: Autores

$\mathrm{Al}$ observar la tabla 1 , se identifica que se puede realizar variantes para la fabricación, considerando así la mejor resistencia que es de nuestro modelo 3 pero para realizar el mecanizado se lo raizará en una materia ASTM A36. Considerando esto se fabrica las cuchillas y se evidencia problemáticas, como es el desgates de las cuchillas y eje, etc. ver figura 7.

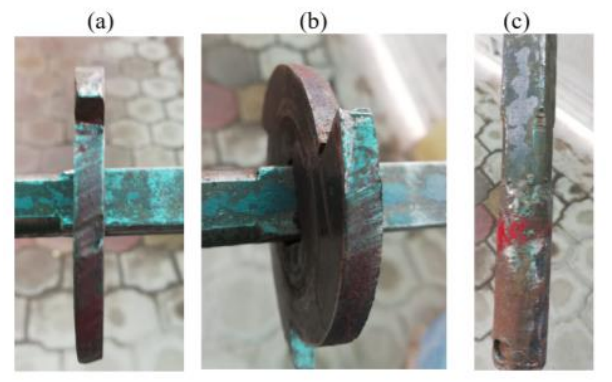

Figura 1. Desgaste en cuchillas y ejes. (a) Desgate por picadura en el cuerpo de cuchilla. (b) Pérdida de filos y despunte. (c) Agrietamiento y picadura de ejes. Fuente: Autores

\section{Conclusiones.}

- La cuchilla con mayores prestaciones es la de tipo C, el material de construcción se puede utilizar acero A36, 1045 o SAE S1 con base de tungsteno la diferencia será representada en la durabilidad y en los costos de mecanizado.

- Basados en economizar los costos de fabricación se puede realizar combinaciones de diseños y materiales con la finalidad de abaratar costos, es así que se recomienda realizar el modelo "C" en un material SAE 1045 o A 36 que prestara las mismas condiciones de trabajo que los realizados con el SAE S1.

- Al trabajar con cuchillas realizadas con A36 se evidencia un desgate prematuro, pero no considerable de las cuchillas, este desgaste no afecta en el funcionamiento de la maquina picadora. 
ISSN: 2600-5859

- La cuchilla al estar sometido a esfuerzos fluctuantes, necesariamente debe presentar una mayor resistencia en las zonas de impacto, por lo que es necesario realizar tratamientos térmicos en las zonas de mayor reincidencia de esfuerzos.

- Los neumáticos reciclados mediante trituración, pueden reutilizarse de manera confiable en materiales compuestos con la finalidad de mejorar las propiedades mecánicas como es el caso de las capas asfálticas, así como también al reutilizar este material se tendrá beneficios ambientales debido a que el residuo se valoriza y solucionará la problemática por mala disposición final de ellos.

\section{Referencias bibliográficas.}

Alejandre, J. G. (Septiembre de 2014). Fundacion vida social. Obtenido de http://www.vidasostenible.org/informes/revolucion-en-reciclaje-de-neumaticos/

American Psychological Association. (2010). Manual de Publicaciones de la American Psychological Association (6 ed.). (M. G. Frías, Trad.) México, México: El Manual Moderno.

Arroyave, G. J. (14 de febrero de 2017). Aplicaciones de caucho reciclado: Una revisión de la Literatura. Obtenido de http://www.redalyc.org/articulo.oa?id=91150559002

C. Flores, S. R. (diciembre de 2014). Revista de la Asociación Latinoamericana de Control de Calidad, Patología y Recuperación de la Construcción. Obtenido de https://www.redalyc.org/pdf/4276/427639593005.pdf

Casas., $\quad$ N. $\quad$ K. (2015). Scribd. Obtenido de https://es.scribd.com/document/293374074/Articulocientifico?fbclid=IwAR0vuHLA3M3sG-sL9MdMXAzGTfGa6O91ATMzAyMxRKzz2CBco0Pue9xCLQ

Castelu, M. D. (18 de 7 de 2017). Revista tecnologica . Obtenido de http://www.revistasbolivianas.org.bo/pdf/rtft/v13n19/v13n19_a02.pdf?fbclid=IwAR 3W-6ZshjwNscBVCAjNJnDtK54p6ZC3d2jk_wTZ6wht7qR2K40KzOifGMc

ChimborazoAzogue, L. E. (2017). Obtenido de http://repositorio.utn.edu.ec/bitstream/123456789/7150/2/ART\%C3\%8DCULO.pdf ?fbclid=IwAR2s_kH3f-EDGZ1YGb8Rq8psBzz3fZXQvZPEYqEMo6JSHcplQemDT0zM2U

Coello- Velázquez, A. L., \& Castellanos- Perez, L. O. (13 de Septiembre de 2017). Mineria y Geología. Obtenido de Mineria y Geología: file://C:/Users/Acer/Downloads/art\%C3\%ADculo_redalyc_223553249001.pdf 
ISSN: 2600-5859

WWW.concienciadigital.org

Vol. 3, N¹.1, p. 29-43, febrero, 2020

Coello-Velázquez, A. L., Hechavarría-Pérez, J. R., \& Castellanos-Pérez, L. (Diciembre de 2017). Modelación matemática de la fragmentación en. Obtenido de http://www.redalyc.org/articulo.oa?id=223553249001

E. Vaquera Gonzalez, S. V. (2018). Revista Ciencia, Ingeniería y Desarrollo Tec Lerd. Obtenido de http://revistacid.itslerdo.edu.mx/coninci2018/CID-035\%20R.pdf

FINA, M. T. (s.f.). Repositorio digital de la universidad tecnica del norte. Obtenido de C:IUsersILUIS ANDRES\Desktop\ARTÍCULO.pdf

Gabriel Jaime Peláez Arroyave, S. M. (14 de Febrero de 2017). Aplicaciones de caucho reciclado: una revision de literatura. Obtenido de Ciencia e ingeniería Neogranadina, vol. 27: http://www.scielo.org.co/pdf/cein/v27n2/0124-8170-cein-27-0200027.pdf?fbclid=IwAR0D-

cGxLKY5rpc_nF39S_itgrlrLN9xHWeFTOYek0E7UfNGbhbsTeEgXYY

Guerrero, E. J. (agosto de 2018). Estudiante de Ingeniería Civil - Universidad Politécnica Salesiana.

Obtenido

de https://dspace.ups.edu.ec/bitstream/123456789/16054/1/UPS-ST003778.pdf

José Ramón Hechavarría-Pérez, A. L.-V.-P.-A. (31 de julio de 2015). Redalyc. Obtenido de http://www.redalyc.org/articulo.oa?id=49642141012\&fbclid=IwAR0ilmME7QY27 JHE40FwLuPy1Bfyhnn2S5ZQhH1VQjS0VoaA0ekzxWzSF8g

MSc., M. F. (2017). MÁQUINA TRITURADORA DE CAUCHO PARA LA OBTENCIÓN DE GRANULOMETRÍA FINA.

Nisbeth, R. G. (2008). Diseño en ingeniería mecánica de Shigley. Mexico: A Subsidiary of The McGraw-Hill Companies, Inc.

Peláez., G. J., \& Giraldo., D. H. (28 de Septiembre de 2017). Ciencia e Ingenieria Neogranadina. Obtenido de Ciencia e Ingenieria Neogranadina: file:///C:/Users/Acer/Downloads/Dialnet-AplicacionesDeCauchoReciclado5836967\%20(1).pdf

Pelizzoni, J. L., Fumagalli, S. A., Quarleri, R. B., \& Raùl, E. (2013). AVANCES DE DISEÑO DE PROTOTIPO DE EQUIPO DE LA EXPLOTACIÒN MINERA. Obtenido de http://www.edutecne.utn.edu.ar/coini_2013/trabajos/COA22_TC.pdf?fbclid=IwAR 2wIcwz0CdxmWGg1BYBbDReyOaGlObd74M4UUiL8DslT-G8YYl-DicRlrc

Roberto Pérez Aparicio, L. S. (Enero de 2018). Departamento de Desarrollo de Mercados y Nuevas Aplicaciones. Obtenido de https://www.signus.es/wpcontent/uploads/2018/04/reciclado-de-neumaticos-transformacion-de-un-residuo- 
ISSN: 2600-5859

www.concienciadigital.org

en-un-recurso.pdf?fbclid=IwAR2_8HdKDcyAG6-

Vol. 3, N¹.1, p. 29-43, febrero, 2020

3OXPgTYWv97yutABFvQW2Ht1Pnn8m90Ix9HPycpp8CNw

Roberto Pérez Aparicio, L. S. (enero de 2018). Revista de plasticos modernos. Obtenido de https://www.signus.es/wp-content/uploads/2018/04/reciclado-de-neumaticostransformacion-de-un-residuo-en-un-recurso.pdf

Urrego Yepes, W., \& Cardona Vazquez, N. (2 de Julio de 2017). Redalyc. Obtenido de Redalyc: http://www.redalyc.org/jatsRepo/4962/496254902 
ISSN: 2600-5859

PARA CITAR EL ARTÍCULO INDEXADO.

Moyano Arévalo, J. R., Naranjo Vargas, E. M., \& Tenicota García, A. G. (2020). Diseño y selección de cuchillas para el proceso de trituración de neumáticos y análisis de costos de fabricación . ConcienciaDigital, 3(1.1), 29-43. https://doi.org/10.33262/concienciadigital.v3i1.1.1130

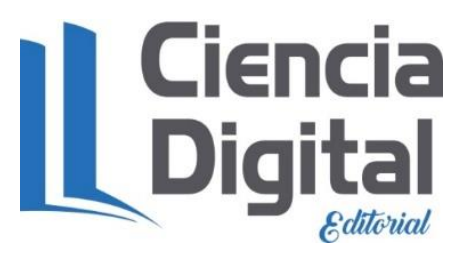

El artículo que se publica es de exclusiva responsabilidad de los autores y no necesariamente reflejan el pensamiento de la Revista Conciencia Digital.

El artículo queda en propiedad de la revista y, por tanto, su publicación parcial y/o total en otro medio tiene que ser autorizado por el director de la Revista Conciencia Digital.

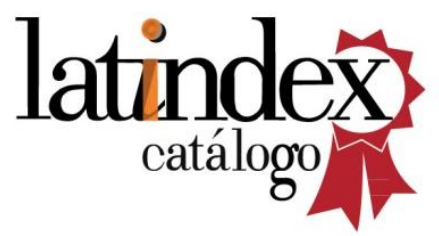

